\title{
The nature of Petschek-type reconnection
}

\author{
T. G. Forbes
}

Institute for the Study of Earth, Oceans, and Space, University of New Hampshire, Durham, NH 03824, U.S.A.

(Received July 20, 2000; Revised November 27, 2000; Accepted November 27, 2000)

\begin{abstract}
It is not always appreciated that Petschek's reconnection mechanism is a particular solution of the MHD equations which applies only when special conditions are met. Specifically, it requires that the flow into the reconnection region be set up spontaneously without external forcing. This condition is satisfied when reconnection in a simple current sheet is initiated by enhancing the resistivity in a localized region. Such a process disrupts the current sheet and launches slow-mode waves which steepen into nearly switch-off shocks of the type predicted by Petschek. As these shocks propagate outwards, the current sheet reforms at the original point of the disturbance, and a quasisteady Petschek-like configuration is set up. Syrovatskii-like configurations which force reconnection by driving a flow toward an initially current-free, orthogonal x-point are less likely to satisfy the conditions required for Petschek-type reconnection.
\end{abstract}

\section{Petschek-Type Solutions}

Petschek's (1964) solution for reconnection is a novel extension of an earlier solution by Sweet (1958) and Parker (1957) for reconnection in a thin current sheet. Both Sweet and Parker assumed that the length of the current sheet was the same as the scale size, $L_{e}$, of the global field. In the Sweet-Parker solution, the Alfvén Mach number, $M_{A e}$, of the plasma flowing into the sheet is related to the magnetic Reynolds number, $R_{m e}$, by

$$
M_{A e}=R_{m e}^{-1 / 2},
$$

where $R_{m e}=L_{e} V_{A e} / \eta, V_{A e}$ is the Alfvén speed in the inflow region, and $\eta$ is the magnetic diffusivity of the plasma. In astrophysical plasmas $R_{m e}$ is typically $10^{6}$ to $10^{12}$, so Sweet-Parker-type reconnection is very slow $\left(M_{A e} \approx 10^{-3}\right.$ to $10^{-6}$ ) compared to the rates needed to explain the rapid release of magnetic energy which occurs in phenomena such as solar flares and magnetospheric substorms $\left(M_{A e} \approx 0.1\right)$.

Petschek's method for speeding up the Sweet-Parker process was to encase it in an external magnetic field so that the length, $L$, of the current sheet would be much smaller than the global scale length, $L_{e}$. In Petschek's configuration the diffusion region current sheet occupies only a small central location, and most of the conversion of magnetic energy into heat and bulk kinetic energy occurs at four standing slowmode shocks attached to the corners of the diffusion region as shown in Fig. 1.

Petschek also assumed that the magnetic field in the inflow region was current free. This assumption, together with the trapezoidal shape of the inflow region created by the slow shocks, leads to a logarithmic decrease of the magnetic field as the inflowing plasma approaches the Sweet-Parker current sheet. This variation of the field leads in turn to

Copy right (C) The Society of Geomagnetism and Earth, Planetary and Space Sciences (SGEPSS); The Seismological Society of Japan; The Volcanological Society of Japan; The Geodetic Society of Japan; The Japanese Society for Planetary Sciences.
Petschek's famous formula for the maximum reconnection rate

$$
M_{A e}=\pi /\left(8 \ln R_{m e}\right),
$$

where $R_{m e}$ and $V_{A e}$ are the magnetic Reynolds number and Alfvén speed in the region far upstream of the current sheet. Because of its logarithmic dependence on $R_{m e}$, the Petschek reconnection rate is many orders of magnitude greater than the Sweet-Parker rate, and for most space and laboratory applications Petschek's formula predicts that $M_{A e} \approx 10^{-1}$ to $10^{-2}$.

Petschek's model incorporates the Sweet-Parker model in order to describe the flow of plasma and fields in the diffusion region. Because the Sweet-Parker model only gives the average properties of the diffusion region, such as its length and thickness, no detailed matching is possible between the flows in the diffusion region and the flows in the external region outside. This lack of detailed matching is sometimes misunderstood to mean that there is no matching at all, but in fact the average properties of the diffusion region are rigorously matched to the external region (see Vasyliunas, 1975).

The reconnection solution developed by Petschek in 1964 is historically important for two reasons. First, it demonstrated the possibility that the rate of reconnection could be quite fast even in plasmas where magnetic diffusion is normally negligible. Second, it introduced the idea that slowmode shocks could play a fundamental role in reconnection. Following its introduction there was a tendency to apply Petschek's solution as a universal solution to any reconnection problem, but with the advent of numerical simulations it soon became apparent that such universal application is unwarranted. Thus the question has arisen as to what kind of configurations are likely to produce Petschek-type reconnection.

Although it was originally obtained using the steady-state MHD equations, Petschek's solution can be understood as 


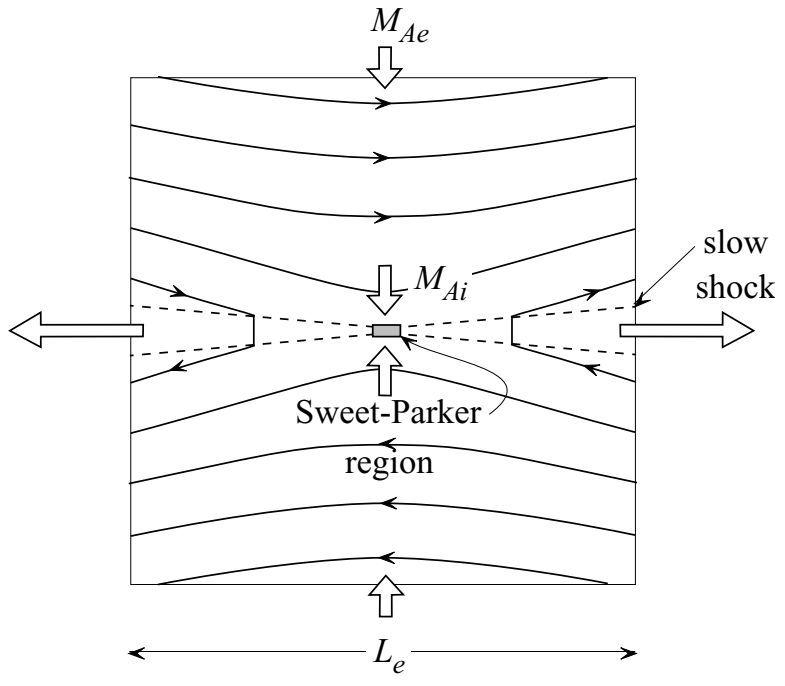

Fig. 1. Magnetic field configuration of Petschek's solution. The small rectangle in the center indicates the short current sheet corresponding to the Sweet-Parker region. The dashed lines are the four slow-mode shocks that radiate outwards from this region. The symbols $M_{A e}$ and $M_{A i}$ stand for the inflow Alfvén Mach numbers at the exterior and interior boundaries of the external region which surrounds the Sweet-Parker region.

the quasi-steady limit of an inherently time-dependent process. The simplest illustration of this process is the disruption of an infinitely long current sheet caused by enhancing the electrical resistivity in a localized region of the sheet. Disruption of the sheet in this manner leads naturally to the formation of outward propagating slow-mode shocks which are an essential feature of Petschek-type reconnection.

Semenov et al. $(1983,1984)$ and Heyn and Semenov (1996) have developed a time-dependent solution for magnetic reconnection which has many of the features of Petschek's steady-state solution. In their solution, reconnection is triggered by an increase in resistivity at a particular location in a current sheet as indicated in Fig. 2(a). The onset of reconnection in this localized region launches both fast and slow magnetoacoustic waves into the plasma (Fig. 2(b)). In the incompressible version of the theory (Biernat and Heyn, 1987; Rijnbeek and Semenov, 1993), the fast-mode waves propagate outwards instantaneously and set up an inflow towards the x-point.

As in the steady-state Petschek theory, the inflow near the $\mathrm{x}$-point is supermagnetosonic with respect to the slow-mode wave speed, so slow-mode-shocks are formed. However, the shocks are curved and now form the trailing boundary of a tear-drop shaped outflow region as shown in Fig. 2(c). The leading boundary of this region is formed by a pair of intermediate waves, or shocks, which connect to the trailing slow-mode shocks. In the incompressible limit, the speed at which the outflow disturbance propagates along the current sheet is just $V_{A e}$, the ambient Alfvén speed, so the external scale-length $\left(L_{e}\right)$ of the system continuously increases with time, $t$, as

$$
L_{e}=V_{A e} t
$$

Also, as in the steady-state Petschek solution, the inflow is current-free to first order. However, this is no longer an arbi- trary assumption, but a requirement which follows naturally from the form of the initial state. At $t=0$, there is no current in the region outside the sheet, so any current which develops there must be set up by waves propagating outwards from the initial reconnection site if there are no external forces driving the reconnection. Fast-mode waves easily propagate upstream, but slow-mode waves have a more difficult time because their wave speed is zero in the direction perpendicular to the field. Once the inflow into the reconnection site becomes well developed, the region upstream of the slow-mode shock is everywhere supermagnetosonic with respect to the slow-mode wave speed.

The inability of slow-mode waves to travel upstream of the slow-mode shocks constrains the current density to be zero in the upstream region to at least first order in the expansion. We can see the reason for this by looking at the momentum equation:

$$
\rho[(\partial \mathbf{u} / \partial t)+(\mathbf{u} \cdot \nabla) \mathbf{u}]=-\nabla p+(\mathbf{j} \times \mathbf{B}) / c,
$$

where $\rho$ is the density, $\mathbf{u}$ is the bulk flow, $p$ is the gas pressure, $\mathbf{j}$ is the current density, $\mathbf{B}$ is the magnetic field, and $c$ is the speed of light. In the region where a quasi-steady flow has been established, the inertial terms $\rho(\partial \mathbf{u} / \partial \mathbf{t})$ and $\rho(\mathbf{u} \cdot \nabla \mathbf{u})$ are negligible. Therefore, to lowest order, the momentum equation is just $\nabla p=(\mathbf{j} \times \mathbf{B}) / c$. Since this is the equation describing a static equilibrium, variations in $p$ and $B$ across field lines must be in the opposite sense, except for the special case when $j=0$. Variations of this sort can only be created by slow-mode waves, but such waves can only reach the inflow region from external sources because the inflow is supermagnetosonic with respect to the slow-mode speed. Consequently, in the absence of any external driving of the flow, the only possible solution is $j=0$ to lowest order. Thus, Petschek's solution corresponds to an undriven form of reconnection.

The solution of Semenov et al. $(1983,1984)$ is set up in much the same manner as Petschek's. In the inflow region one expands the equations in terms of the smallness parameter $\epsilon$ defined as

$$
\epsilon(t)=c E(t) /\left(V_{A e} B_{e}\right),
$$

where $E(t)$ is the electric field at the x-point and $B_{e}$ is the ambient field outside the sheet. The parameter $\epsilon$ plays the same role as the Alfvén Mach number $\left(M_{A e}\right)$ of the steadystate theory, and the expansion assumes $\epsilon \ll 1$. Another restriction on $E$ is that it must change slowly with respect to time, so that the evolution of the diffusion region at the $\mathrm{x}$-point is quasi-steady. This restriction, combined with the fact that $\epsilon \ll 1$, makes the outflow region a thin layer and permits solutions of the MHD equations by using boundary layer theory.

The coupling between the diffusion region and the idealMHD dynamics of the external region is done in the same manner as for the steady-state Petschek solution, namely by matching the external solution to the Sweet-Parker relations. A time-dependent external solution is first obtained by treating the diffusion region as a point for which the electric field (i.e. the time-dependent reconnection rate) is an arbitrary function of time. After the external solution is obtained, a 
(a)

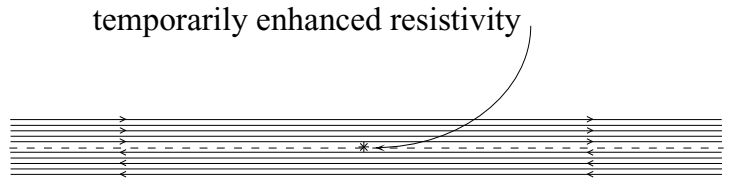

(b)

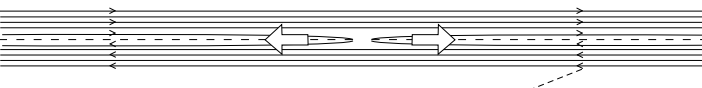

Petschek-like region

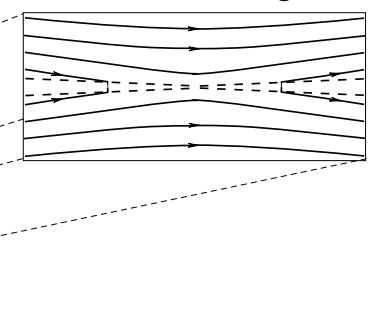

(c)

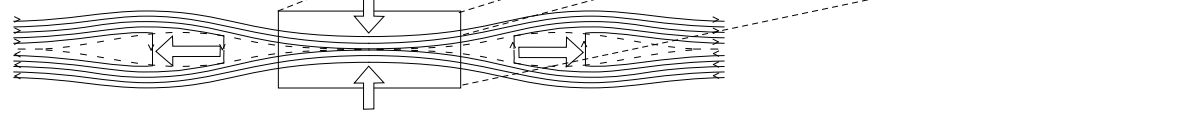

Fig. 2. Evolution of the magnetic field configuration in the time-dependent solution of Semenov et al. (1984). Reconnection is initiated by enhancing the resistivity in a localized region of a simple current sheet, and the outflow from the $\mathrm{x}$-point creates two regions which propagate outwards along the sheet in opposite directions. The leading edges of these flow regions are bounded by intermediate waves, or shocks, and the trailing edges are bounded by slow mode shocks. The reformation of the current sheet at the initial point of disturbance leads to a quasi-steady Petschek-like configuration.

Petschek-like analysis is done in the vicinity of the diffusion region to relate the variaton of the electric field to the variation of the resistivity with time, but the procedure is only valid if the variations are sufficiently slow in time to be considered quasi-steady. Such a procedure leads to the result that the normalized electric field varies as

$$
\epsilon(t) \approx \pi /\left[4 \ln R_{m e}(t)\right]
$$

where $R_{m e}(t)=V_{A e} L_{e}(t) / \eta=V_{A e}^{2} t / \eta$, and $\eta$ is the value of the diffusivity after its initial increase. Except for a factor of 2 , this is the same as Petschek's result but with the magnetic Reynolds number, $R_{m e}$, now a function of time. A more formal matching between the external and diffusion regions has been published recently by Erkaev et al. (2000).

It may seem paradoxical that Petschek's steady-state formula should still apply to a highly time-dependent configuration involving waves propagating at the Alfvén speed, but the evolution of the field and flow in the vicinity of the diffusion region is quite slow. This slowness is evident from the formula for the length, $L$, of the diffusion region current sheet

$$
L=L_{e} R_{m e}^{-1} \epsilon^{-2}=\left[\eta / V_{A e}\right]\left[4 \pi^{-1} \ln \left(V_{A e}^{2} t / \eta\right)\right]^{2}
$$

which depends only logarithmically on time. Thus, the evolution of the flow and field in the diffusion region can be considered quasi-steady as Petschek originally assumed.

There are several numerical simulations which exhibit the behavior predicted by the time-dependent Petschek theory (e.g. Ugai and Tsuda, 1977; Ugai, 1984, 1988, 1995; Scholer, 1989; Schumacher and Kliem, 1996). These simulations trigger reconnection in a simple current sheet by enhancing the diffusivity at a particular location, and they use open boundary conditions which allow wave disturbances to exit the boundaries of the numerical domain without reflection.

\section{Syrovatskii-Type Solutions}

A different way to approach the reconnection process in two dimensions is to ask what happens if flows are driven to- wards an initially current-free x-point as illustrated in Fig. 3. In an ideal, low $\beta$ plasma an infinitely thin current sheet forms, whose length grows with time. The mathematical description of such a nonreconnecting sheet was first obtained by Green (1965) and later generalized by Syrovatskii (1971) and Somov (1992). Syrovatskii (1971) pointed out that the ideal solution provides an approximate description of the nonideal reconnection process, so long as the rate of reconnection is slow (i.e. $M_{A} \ll 1$ ). However, the actual formula for the reconnection rate was first determined numerically by Biskamp (1986) and later analytically by Strachan and Priest (1994).

The magnetic field for the Syrovatskii-type solution found by Strachan and Priest (1994) has the form

$$
B_{y}+i B_{x}=B_{o}\left[(z / L)^{2}-1\right]^{1 / 2}-i \alpha B_{o}\left(y / L_{e}\right),
$$

where $z=x+i y, B_{o}$ is a constant, $L$ is the length of the current sheet, and $L_{e}$ is the global scale length of a rectangular domain as shown in Fig. 4. The parameter $\alpha$ gives the strength of the uniform current density in the inflow region, and it is determined from the normal component of the magnetic field imposed at the inflow boundary.

To obtain the reconnection rate, Strachan and Priest (1994) matched the external field region prescribed by the above equation to the Sweet-Parker solution in much the same way as Petschek did for his solution. However, the different form of the external field causes the resulting solution to behave very differently from the one found by Petschek. Whereas Petschek found that the maximum rate occurs when the central diffusion region current sheet is as short as possible, Strachan and Priest (1994) found that the maximum rate occurs when the current sheet is as long as possible. The maximum length occurs when $L=L_{e}$, and the corresponding maximum rate (for a square domain) is

$$
M_{A e}=\left[2 /\left(5^{1 / 2}-1\right)\right]^{3 / 4} R_{m e}^{-1 / 2}=1.43 R_{m e}^{-1 / 2}
$$

which is the same as the Sweet-Parker rate, except for the factor of 1.43 (Priest and Forbes, 1992). 


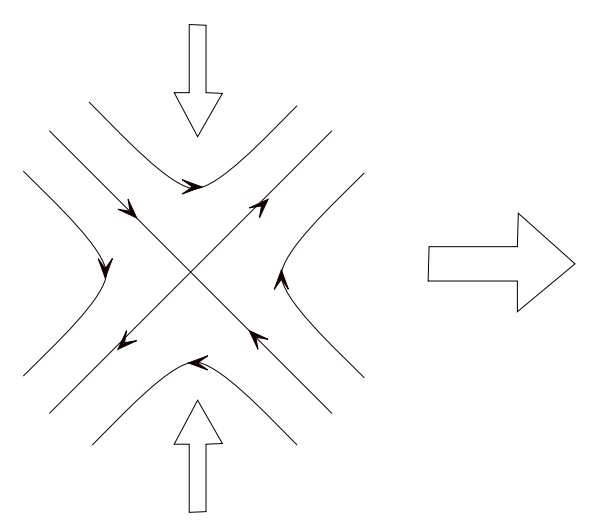

(a)

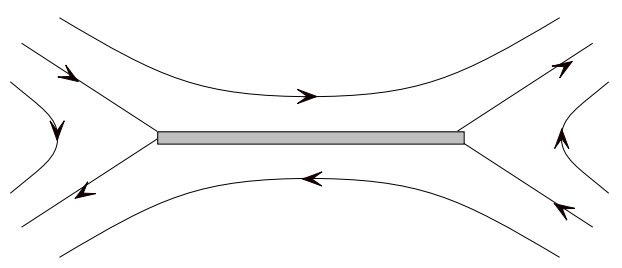

(b)

Fig. 3. (a) Syrovatskii-like reconnection solutions are based on a current-free x-point configuration which is created by external field sources that are not present in the Petschek configuration. (b) A current sheet is formed at the initially current-free x-point by externally driving flows towards it.

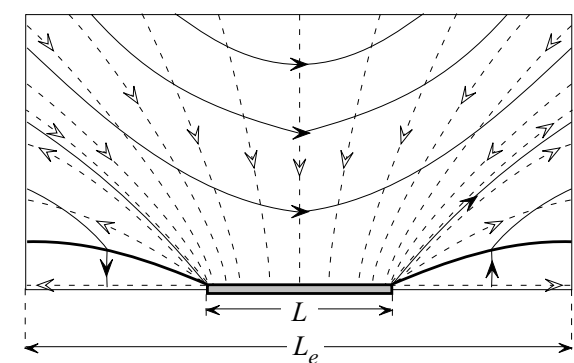

Fig. 4. Magnetic field lines (solid) and streamlines (dashed) for the solution of Strachan and Priest (1994) which is based on a Syrovatskii-type configuration. The rectangle of length $L$ is the Sweet-Parker region which is matched to the external region of length, $L_{e}$, in the same way as in Petschek's solution. The principal difference between this solution and Petschek's is the presence of the nonuniform background field created by external sources.

From the Strachan and Priest (1994) analysis it is evident that Syrovatskii-type solutions scale differently with magnetic Reynolds number than Petschek's solution do because of the orthogonal background field (cf. Fig. 3(a)). This field is generated by external current sources which exist independently of the field produced by the current sheet. Such external sources do not exist in Petschek's solution because the only sources of the field in it are the central current sheet and the slow mode shocks. The presence of the background field alters the coupling between the inflow magnetic field and flow, so that the current sheet increases in length with increasing $M_{A e}$.

The Sweet-Parker type scaling in the Syrovatskii-type configurations is a natural consequence of the reversal in the relation between the length of the current sheet and $M_{A e}$. The reversal causes the maximum rate to occur when the length, $L$, of current sheet is the same as the global scale, $L_{e}$, and this is one of the principal assumptions of the SweetParker analysis. Consequently, once $L$ is set equal to $L_{e}$, Sweet-Parker scaling is to be expected.

Several numerical simulations (Sato, 1983; Biskamp, 1986; Lee and Fu, 1986; Scholer, 1989; Yan et al., 1992; Jin and Ip, 1991) have been carried out in an attempt to verify the steady-state solution found by Petschek (1964), but none of these simulations have been able to replicate the scaling results predicted by Petschek's solution as long as the resistivity is kept uniform and constant. For example, Biskamp (1986) found that both the length and the thickness of the diffusion region increase as $M_{A e}$ or $R_{m e}$ increases, as shown in Fig. 5. This behavior completely contradicts Petschek's prediction. Thus, Biskamp realized that his numerical experiments, as well as those of other researchers, had not found the scaling predicted by Petschek's model.

As Fig. 6 shows, the scaling for the maximum reconnection rate found by Biskamp is essentially the same as predicted by the Strachan-Priest solution. When adjusted for the non-square box of Biskamp's simulations, the StrachanPriest theory gives $M_{A e}=3.42 R_{m e}^{-1 / 2}$, and, as Fig. 6 shows, this is approximately the same result found by Biskamp (1986).

\section{Generalization of Petschek's Solution}

Although Petschek assumed that the current density, $j$, in the inflow region was zero to first order, it is not actually necessary to make such an assumption in order to obtain a solution. More generally, $j$, can be nonzero to first order in the expansion of the inflow equations, so that the inflow magnetic field is no longer determined by solving Laplace's equation $\left(\nabla^{2} A=0\right)$ for the vector potential, $A$, but by solving Poisson's equation $\left(\nabla^{2} A=-4 \pi j / c\right)$ instead. The relaxation of the assumption that $j$ is zero introduces an additional degree of freedom so that there is now a family of solutions (Priest and Forbes, 1986).

These solutions can be summarized in terms of the relation between the internal Alfvén Mach number, $M_{A i}$, at the entrance to the diffusion region as indicated in Fig. 1, and $M_{A e}$, the Alfvén Mach number at the exterior inflow boundary. The relation is

$$
\begin{aligned}
M_{A e}^{1 / 2} M_{A i}^{-1 / 2}= & 1-4 M_{A e} \pi^{-1}(1-b)[0.834 \\
& \left.-\ln \tan \left(\pi R_{m e}^{-1} M_{A e}^{-1 / 2} M_{A i}^{-3 / 2} / 4\right)\right]
\end{aligned}
$$

where $b$ is a constant that is determined by the choice of 


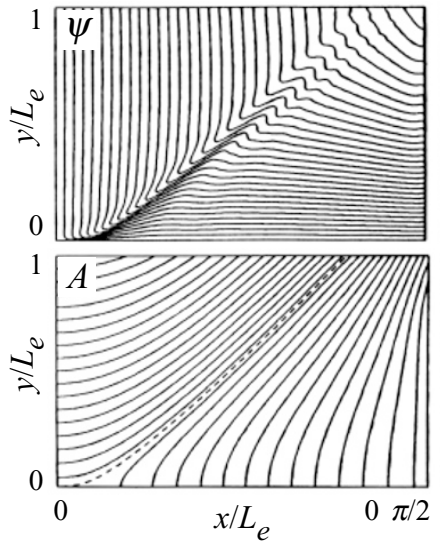

(a)
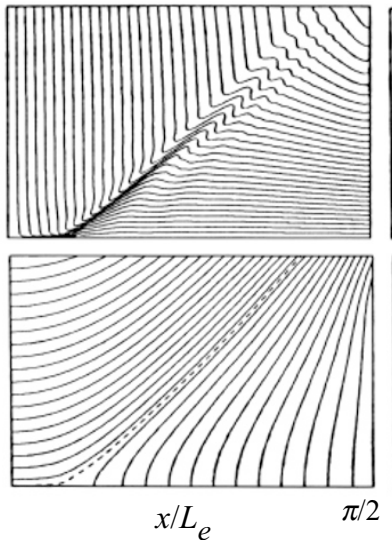

(b)

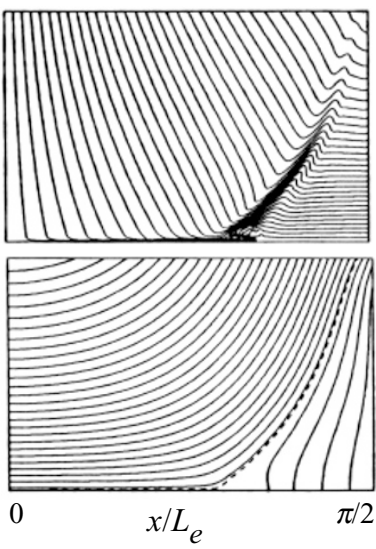

(c)

Fig. 5. Streamlines (top) and magnetic field lines (bottom) in the numerical simulation of Biskamp (1986) for $M_{A e}=0.042$ and (a) $R_{m e}=1$, 746, (b) $R_{m e}=3,492$, and (c) $R_{m e}=6,984$. Only the upper left quadrant is shown, and the x-point is located at the lower-right corner. As $R_{m e}$ is increased, the current grows longer. Such behavior is opposite to that predicted by Petschek's solution.

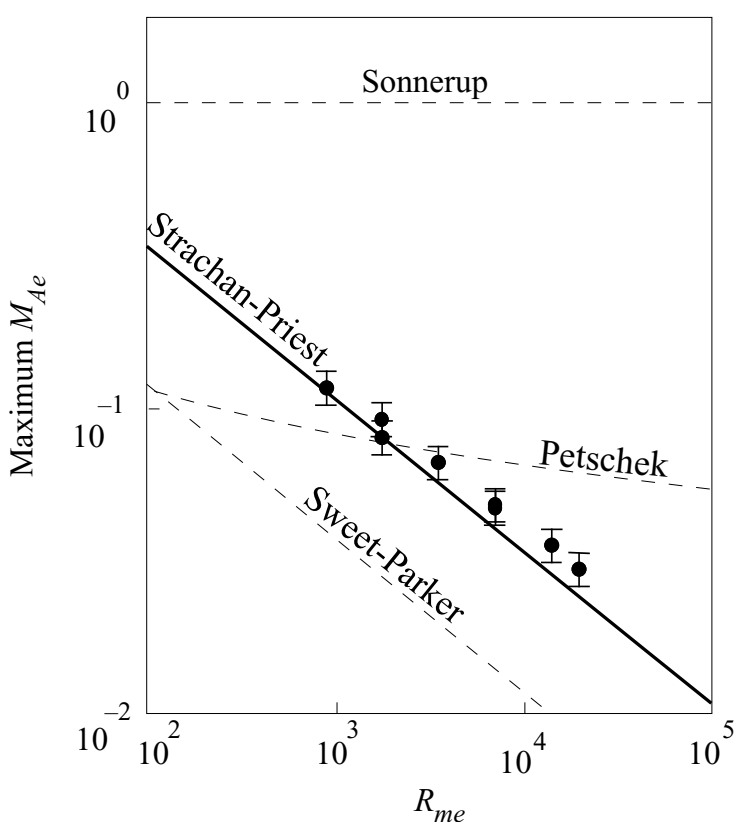

Fig. 6. The variation of the maximum reconnection rate with magnetic Reynolds number predicted by the analytical theory of Strachan-Priest (dark solid line). The scaling results from Biskamp's (1986) numerical simulation are indicated by the dots with error bars. The agreement between the two seems especially impressive when it is realized that there are no free parameters in the analytical theory once it is matched to the boundary conditions used in the simulation. Also shown by the dashed curves are the solutions of Sweet (1958) and Parker (1957), Petschek (1964), and Sonnerup (1970).

the inflow boundary conditions. The relation is plotted in Fig. 7 for $R_{m e}=500$ for various values of $b$. When $b=0$, Petschek's solution is obtained as shown in Fig. 1, and when $b=1$, a solution equivalent to that of Sonnerup (1970) is obtained.

As $b$ increases beyond 1, a flux-pile-up regime occurs where the magnetic field increases as the diffusion region current sheet is approached. For very strong flux-pile-up with $b \gg 1$, the flow approaches the MHD stagnation-point

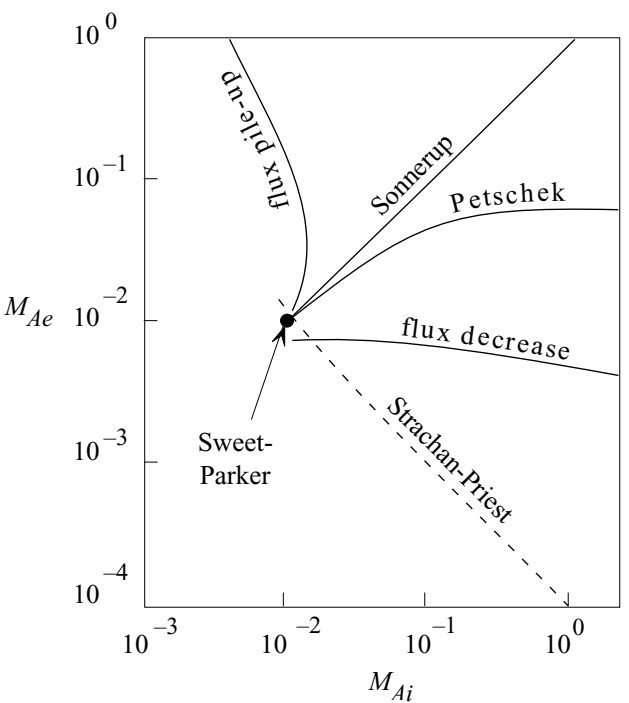

Fig. 7. The external Alfvén Mach number, $M_{A e}$, versus the internal Alfvén Mach number, $M_{A i}$, (see Fig. 1) for various steady-state theories. The solutions labeled "flux pile-up", "Sonnerup", "Petschek", and "flux decrease" are obtained from the generalized solution of Priest and Forbes (1986) by setting the boundary parameter $b$ in their solution equal to 10 , 1,0 , and -10 , respectively. As $b$ decreases from positive to negative values, the character of the inflow changes from that of a slow-mode expansion, where the gas pressure decreases but the magnetic flux increases (i.e. piles-up), to that of a slow-mode compression, where the gas pressure increases but the magnetic flux decreases. Note that the Syrovatskii-like solution of Strachan and Priest (dashed line) behaves similarly to the flux decrease solution for $b=-10$ (after Priest and Forbes, 2000).

flow solution found by Parker (1973). The flux pile-up solutions appear to be very fast since formally, $M_{A e}$ can be as large as one. However, Litvinenko and Craig (1999) have shown that $M_{A e}$ is actually limited to rather small values unless the plasma $\beta$ is very much greater than unity. For a low $\beta$ plasma the fastest rate occurs for $b=0$, which is Petschek's solution. For $b<0$ the magnetic field decreases as the sheet is approached, and this is qualitatively 
the same behavior, as occurs in the Syrovatskii-type solutions of Biskamp (1986) and Strachan and Priest (1994).

Vasyliunas (1975) was the first to point out that the difference between the behavior predicted by Petschek's solution and other reconnection solutions is related to the behavior of the gas and magnetic pressures in the inflow region. The inflow can be characterized as undergoing a compression or an expansion depending on whether the gas pressure increases or decreases as the plasma flows in towards the x-point. These compressions or expansions can further be characterized as being of the fast-mode type or the slow-mode type, depending on whether the magnetic pressure changes in the same sense as the gas pressure (fast-mode type) or in the opposite sense (slow-mode type). This way of characterizing the inflow is still valid even if one assumes, as Petschek did, that the plasma behaves incompressibly. (By the way, a compressible version of Petschek's theory may be found in Soward and Priest (1982) for those who are interested in the effects of compressibility.)

Here we illustrate Vasyliunas's classification scheme using the generalized Petschek solution, but the scheme can be applied to any reconnection solution obtained from the MHD equations. For $b=0$ (i.e. Petschek's solution) the gas pressure is uniform to second order in the expansion in $M_{A e}$, so that to this order, the plasma is neither compressed nor rarefied as it flows towards the x-point. However, for all the solutions with $b<0$, the plasma undergoes a slowmode compression, while for all the solutions with $b>0$ it undergoes a slow-mode expansion. Thus, as $b$ increases from negative to positive values, the inflow turns from being converging (producing a slow-mode compression) to being diverging (producing a slow-mode expansion). The special significance of Petschek's solution is that it is the only one which is not forced by external driving of the flow, and therefore, the only one without any change in the gas pressure to first order.

\section{Conclusions}

There has been a strong tendency in the development of reconnection theory for particular solutions to be championed at the expense of other solutions. For example, when Petschek's solution was introduced in 1964, it was thought by many that it could be universally applied. Later when alternate solutions, such as those of Sonnerup (1970) and Biskamp (1986) were discovered, it was often assumed that only one of these solutions could be right and that the others must, therefore, be wrong. However, the idea that a single universal solution exists which can be applied in any situation is a mathematical impossibility. Solutions of the system of differential equations which governs plasmas necessarily depend on the choice of initial and boundary conditions, and the process of magnetic reconnection does not in of itself impose any specific choice for these conditions.

The important feature of Petschek-type reconnection is that it is not driven by any external forcing. Except for the spontaneous tearing mode, all the other types of known reconnection solutions require an external flow, or force, to drive the reconnection. The realization that Petschek's solution is undriven may explain why it has been so difficult to achieve steady-state Petschek-type reconnection in numer- ical simulations. Simulations which drive the flow at the boundaries, or start with a current free x-point, controvert the basic assumptions of Petschek-type reconnection. On the other hand, simulations which use the onset of enhanced resistivity in a current sheet to produce a Petschek-like configuration can never achieve a truly steady state, although they may achieve a quasi-steady state in the vicinity of the $\mathrm{x}$-point. From this point of view, the boundaries enclosing Petschek's solution are not true physical boundaries, but rather mathematical ones. The conditions at these mathematical boundaries must precisely correspond to the quasisteady conditions set up after the passage of the waves produced by the initial disturbance.

So far, the only way, to set up an exact steady-state Petschek configuration has been to assume a nonuniform resistivity in a region whose length is tailored to match that required by Petschek's solution (Yan et al., 1992). It may be that an exact steady-state solution with uniform resistivity is inherently unstable, but even if this is true, the fundamental predictions of Petschek's solution remain valid. The first is that the reconnection process can produce slowmode shocks which can be more important for the conversion of magnetic energy than the Sweet-Parker diffusion region. The second is that the reconnection rate can, at least in principle, be quite fast $\left(M_{A e}>0.01\right)$ even when the magnetic Reynolds is very large $\left(R_{m e}>10^{10}\right)$.

Acknowledgments. The author thanks the organizers of the MR2000 for their invitation to present this work at the University of Tokyo 2000 Symposium. This work was supported by NASA grants NAG5-9614 and NAG5-1479 to the University of New Hampshire.

\section{References}

Biernat, H. K. and M. F. Heyn, Unsteady Petschek reconnection, J. Geophys. Res., 92, 3392-3396, 1987.

Biskamp, D., Magnetic reconnection via current sheets, Phys. Fluids, 29, 1520-1531, 1986.

Erkaev, N. V., V. S. Semenov, and F. Jamitzky, Reconnection rate for the inhomogeneous resistivity Petschek model, Phys. Rev. Lett., 84, 14551458, 2000.

Green, R. M., Modes of annihilation and reconnection of magnetic fields, in Solar and Stellar Magnetic Fields, edited by R. Lüst, pp. 398-404, North-Holland Publishing Co., Amsterdam, 1965.

Heyn, M. F. and V. S. Semenov, Rapid reconnection in compressible plasma, Phys. Plasmas, 3, 2725-2741, 1996.

Jin, S.-P. and W.-H. Ip, Two-dimensional compressible magnetohydrodynamic simulation of the driven reconnection process, Phys. Fluids $B, \mathbf{3}$, 1927-1936, 1991

Lee, L. C. and Z. F. Fu, Multiple x-line reconnection, 1. A criterion for the transition from a single $\mathrm{x}$-line to a multiple $\mathrm{x}$-line reconnection, $J$. Geophys. Res., 91, 6807-6815, 1986.

Litvinenko, Y. E. and I. J. D. Craig, Magnetic energy release in flux pile-up merging, Solar Phys., 189, 315-329, 1999.

Parker, E. N., Sweet's mechanism for merging magnetic fields in conducting fluids, J. Geophys. Res., 62, 509-520, 1957.

Parker, E. N., Comments on the reconnexion rate of magnetic fields, $J$. Plasma Phys., 9, 49-63, 1973.

Petschek, H. E., Magnetic field annihilation, in The Physics of Solar Flares, edited by W. N. Hess, pp. 425-439, NASA, SP-50, 1964.

Priest, E. R. and T. G. Forbes, New models for fast steady-state magnetic reconnection, J. Geophys. Res., 91, 5579-5588, 1986.

Priest, E. R. and T. G. Forbes, Does fast magnetic reconnection exist?, J. Geophys. Res., 97, 16757-16772, 1992.

Priest, E. R. and T. G. Forbes, Magnetic Reconnection-MHD Theory and Applications, 600 pp., Cambridge Univ. Press, Cambridge, 2000.

Rijnbeek, R. P. and V. S. Semenov, Features of a Petschek-type reconnection model, Trends in Geophys. Res., 2, 247-268, 1993. 
Sato, T., Strong plasma acceleration by slow shocks resulting from magnetic reconnection, J. Plasma Phys., 30, 109-124, 1983.

Scholer, M., Undriven magnetic reconnection in an isolated current sheet, J. Geophys. Res., 94, 8805-8812, 1989.

Schumacher, J. and B. Kliem, Dynamic current sheets with localized anomalous resistivity, Phys. Plasmas, 3, 4703-4711, 1996.

Semenov, V. S., M. F. Heyn, and I. V. Kubyshkin, Reconnection of magnetic field lines in a nonstationary case, Sov. Astron., 27, 660-665, 1983.

Semenov, V. S., E. P. Vasilyev, and A. I. Purovkin, A scheme for the nonsteady reconnection of magnetic lines of force, Geomagnet. Aeronomy (Engl. Transl.), 24, 370-373, 1984.

Somov, B. V., Physical Processes in Solar Flares, 249 pp., Kluwer, Dordrecht, 1992.

Sonnerup, B. U. Ö., Magnetic-field reconnection in a highly conducting incompressible fluid, J. Plasma Phys., 4, 161-174, 1970.

Soward, A. M. and E. R. Priest, Fast magnetic field-line reconnection in a compressible fluid, 1, Coplanar field lines, J. Plasma Phys., 28, 335-367, 1982.

Strachan, N. and E. R. Priest, A general family of nonuniform reconnection models with separatrix jets, Geophys. Astrophys. Fluid Dynamics, 74 245,1994

Sweet, P. A., The neutral point theory of solar flares, in Electromagnetic Phenomenon in Cosmical Physics, edited by B. Lehnert, pp. 123-139,
Cambridge Univ. Press, New York, 1958.

Syrovatskii, S. I., Formation of current sheets in a plasma with a frozenin strong magnetic field, Sov. Phys. JETP (Engl. Transl.), 33, 933-940, 1971.

Ugai, M., Self-consistent development of fast magnetic reconnection with anomalous plasma resistivity, Plasma Phys. Contr. Fusion, 26, 1549, 1984.

Ugai, M., MHD simulations of fast reconnection spontaneously developing in a current sheet, Computer Phys. Communications, 49, 185-192, 1988.

Ugai, M., Computer studies on plasmoid dynamics associated with the spontaneous fast reconnection mechanism, Phys. Plasmas, 2, 33203328, 1995.

Ugai, M. and T. Tsuda, Magnetic field-line reconnexion by localized enhancement of resistivity. I - Evolution in a compressible MHD fluid, $J$. Plasma Phys., 17, 337-356, 1977.

Vasyliunas, V. M., Theoretical models of magnetic field line merging, 1, Rev. Geophys., 13, 303-336, 1975.

Yan, M., L. C. Lee, and E. R. Priest, Fast magnetic reconnection with small shock angles, J. Geophys. Res., 97, 8277-8293, 1992.

T. G. Forbes (e-mail: tgforbes@bluemoon.sr.unh.edu) 